\title{
Theoretical mathematical model of non-invasive flow quantification for future implementation in routine clinical MRI
}

\section{Laura Fanea}

Radiology Department, Cluj County Hospital, str. Clinicilor, nr. 3 - 5, 400006 Cluj-Napoca, Romania.

Accepted 13 October, 2017

\begin{abstract}
Automated push-button medical analysis using quantitative multiparametric standard scales will offer the possibility of efficient fast medical diagnosis in a precise and accurate manner. Magnetic Resonance Imaging (MRI) offers complex geometrico-physicochemical analysis of the physio-anatomy of interest. Most imaging protocols for the quantitative multiparametric MRI have already been implemented clinically, but flow assessment still remains a problem, especially when evaluated without contrast agents. A simple method for flow quantification using MRI without contrast agents is described in this study. The proposed method has the potential of non-invasive flow quantification, produced offline from MRI images acquired in less than one minute.
\end{abstract}

Keywords: MRI, multiparametric medical diagnosis, in vivo flow quantification, medical image processing, non-invasive imaging.

E-mail: laura.fanea@gmail.com.

\section{INTRODUCTION}

Complex geometrico-physicochemical analysis of the physio-anatomy of interest is needed to understand disease mechanisms, detect, stage or assess disease and evaluate efficacy of new or existing therapies (Miller et al., 2014). Among the existing imaging techniques, $\mathrm{MRI}$ offers the most complex multiparametric geometricophysicochemical information of the physio-anatomy of interest. The information includes: lengths or thicknesses (Fanea and Fagan, 2012), areas (Feczko et al., 2009), volumes (Feczko et al., 2009), relaxation times (Fanea and Fagan, 2012; Bojorquez et al., 2017), diffusion coefficients and fractional volumes of the diffusing components detected (Dong et al., 2004; Le Bihan, 2014), magnetization transfer ratios and the protein concentration (Gupta, 2002; Oreja-Guevara et al., 2006; Henkelman et al., 2001), magnetic susceptibility and iron or calcification concentrations (Duyn, 2013; Wang and Liu, 2015; Liu et al., 2015), and shear stiffnesses in elastography (Yin et al., 2016; Mariappan et al., 2010;
Venkatesh and Ehman, 2015; Hiscox et al., 2016). More recently, magnetic resonance spectroscopy has been implemented clinically to analyze the complex biochemical composition of the anatomy imaged (Soares and Law, 2009). All these parameters can be assessed without contrast agents (Fanea and Fagan, 2012; Calamante et al., 1999). Since 1990, several mathematical models have been developed (Fanea et al., 2012; Sourbrone and Buckley, 2013) and implemented in routine clinical MRI for flow quantification using contrast agents for MRI. More recent developments focused on flow quantification using arterial spin labeling techniques for MRI without contrast agents. However, these techniques are still difficult to implement in routine clinical MRI due to their decreased sensitivity (Grade et al., 2015; Amukotuwa et al., 2016; Haller et al., 2016; Calamante et al., 1999). An extremely simple and ready for clinical implementation method of flow quantification using MRI without contrast agents is proposed in this study. 


\section{Mathematical model}

The theoretical model assesses quantitatively flow based on the MRI signal intensity differences between corresponding anatomical regions in control/normal $(\mathrm{C} / \mathrm{N})$ and disease-affected (DA) images. A relative-to-normal global flow coefficient: $f$ is calculated from this model and disease can be detected for $f$ values other than 0 .

If the spin density, $M_{0}$ is assessed from a proton density (PD) image (Brown et al., 2014; Bernstein et al., 2004) and $T_{1}$ and $T_{2}$ of the region analyzed are known or calculated using the scenarios proposed in the literature (Deoni et al., 2005; Fanea and Sfrangeu, 2011), flow can be quantified using two MRI images. One image needs to be acquired from a disease-suspected subject and the other one from a $\mathrm{C} / \mathrm{N}$ subject; unless a standard database already exists for the $\mathrm{C} / \mathrm{N}$ image where flow can be assessed simply by acquiring an MRI image of the DA subject.

\section{METHODOLOGY}

An MRI protocol eliminating or significantly reducing all other effects with exception of the $T_{1}, T_{2}$, and flow can be used to acquire these images. In this situation, the signal intensity changes can be quantified based on the general $T_{1}$ and $T_{2}$ dependent magnetization $(\mathrm{M})$ :

$M=C \times M_{0}$

where $\mathrm{C}$ is the constant determined by the acquisition parameters and the $T_{1}$ and $T_{2}$ relaxation times.

The signal intensity of MRI images depends on the main acquisition parameters used: echo time (TE), repetition time (TR), flip angle (FA) and the physicochemical properties of the region imaged: the PD quantified by $M_{0}$, and the relaxometry quantified by $T_{1}$ and $T_{2}$ (Brown et al., 2014; Bernstein et al., 2004).

If flow differences exist between the two corresponding $\mathrm{C} / \mathrm{N}$ and $\mathrm{DA}$ regions, the difference $(\Delta)$ of $\mathrm{M}$ between these two regions will be greater or less than 0 . In this particular situation, $\Delta$ depends on the: $T_{1}, T_{2}$ relaxation times, and the relative-to-normal global flow effects over the image acquisition time, TR. The relative-to-normal global flow effect can be quantified using the relative-to-normal flow coefficient: $f$. The difference between $M$ in the corresponding $D A$ and $C / N$ regions can be expressed using Equation 1 and the relative-to-normal global flow effect on the initial spin density, $M_{0}$ :

$M^{D A}-M^{C / N}=C^{D A} \times M_{0}^{D A}-C^{C / N} \times M_{0}^{C / N}+f /(\lambda \times T R) \times\left(M_{0}^{D A}-M_{0}{ }^{C / N}\right)$

where $\lambda$ represents the blood-to-tissue partition coefficient. Its values range from 0.77 to 1.04 (Herscovitch and Raichle, 1985), while a more general mean value of $0.9 \mathrm{ml} / \mathrm{g}$ is accepted in many studies (Roberts et al., 1996).

\section{RESULTS}

$M_{0} / M$ and two more abbreviations (AB1) and (AB2) will be used to express $f$ :

$\Delta_{0}=\mathrm{M}_{0}^{\mathrm{DA}}-\mathrm{M}_{0}^{\mathrm{C} / \mathrm{N}}(\mathrm{AB} 1)$

$\Delta=M^{D A}-M^{C / N}(A B 2)$

For simplicity, $\mathrm{M}_{0}{ }^{\mathrm{DA}} / \mathrm{M}_{0}{ }^{\mathrm{C} N \mathrm{~N}}$ can be expressed using (AB1):

$\mathrm{M}_{0}^{\mathrm{C} / \mathrm{N}} \times\left(\mathrm{M}_{0}^{\mathrm{DA}} / \mathrm{M}_{0}^{\mathrm{C} / \mathrm{N}}-1\right)=\Delta_{0}(\mathrm{AB} 1.1)$

$\mathrm{M}_{0}^{\mathrm{DA}} / \mathrm{M}_{0}^{\mathrm{C} / \mathrm{N}}-1=\Delta_{0} / \mathrm{M}_{0}^{\mathrm{C} / \mathrm{N}}(\mathrm{AB} 1.2)$

$\mathrm{M}_{0}{ }^{\mathrm{DA}} / \mathrm{M}_{0}{ }^{\mathrm{C} / \mathrm{N}}=\Delta_{0} / \mathrm{M}_{0}{ }^{\mathrm{CN}}+1(\mathrm{AB} 1.3)$

The expression of $\Delta$ is obtained by introducing Equation 1, (AB1), (AB2), and (AB1.3) in Equation 2:

$\Delta=f /(\lambda \times T R) \times \Delta_{0}+\left(C^{C / N} \times M_{0}^{C / N}\right) \times\left[\left(C^{D A} / C^{C / N}\right) \times\left(M_{0}^{D A} / M_{0}^{C / N}\right)-1\right]$

Rearranging the terms in Equation 3:

$\mathrm{f} /(\lambda \times \mathrm{TR}) \times \Delta_{0}=\Delta-\left(\mathrm{C}^{\mathrm{C} / \mathrm{N}} \times \mathrm{M}_{0}^{\mathrm{C} / \mathrm{N}}\right) \times\left[\left(\mathrm{C}^{\mathrm{DA}} / \mathrm{C}^{\mathrm{C} / \mathrm{N}}\right) \times\left(\Delta_{0} / \mathrm{M}_{0}^{\mathrm{C} / \mathrm{N}}+1\right)-1\right]$ 
f can be expressed in $\mathrm{ml} / \mathrm{g} / \mathrm{ms}$ :

$f=(\lambda \times T R) \times\left\{\Delta / \Delta_{0}-\left(C^{C / N} \times M_{0}^{C / N} / \Delta_{0}\right) \times\left[\left(C^{D A} / C^{C / N}\right) \times\left(\Delta_{0} / M_{0}{ }^{C / N}+1\right)-1\right]\right\}$

$\mathrm{f}_{100 \mathrm{~g}}{ }^{\mathrm{min}}$ can also be introduced to quantify flow in $\mathrm{ml}$ of fluid / $100 \mathrm{~g}$ tissue / $\mathrm{min}$ :

$\mathrm{f}_{100 \mathrm{~g}}{ }^{\mathrm{min}}=\left[10^{\wedge}(-2)\right] / 6 \times \mathrm{f}[\mathrm{ml} / 100 \mathrm{~g} / \mathrm{min}]$

\section{f calculation using MRI images}

The particular expression of $f$ is also presented for two cases involving the most often used MRI imaging acquisition protocols: classical and spoiled gradient echo.

In classical MRI, $M$ depends on the physicochemical parameters of the region imaged: $M_{0}, T_{1}, T_{2}$ and the TE, and TR image acquisition parameters, while for spoiled gradient echo acquisition protocols, FA is also involved, as expressed in Equations 7 and 8 (Brown et al., 2014; Bernstein et al., 2004).

$$
M= \begin{cases}M_{0} \times(1-E 1) \times E 2(7): \text { classical } & \\ M R I \text { acquisition protocols. } & \\ M_{0} \times[\sin F A \times(1-E 1) \times E 2] /(1-E 1 \cos F A)(8): \text { spoiled gradient echo }\end{cases}
$$

The following two abbreviations were used in Equations 7 and 8:

$E 1=\exp \left(-T R / T_{1}\right)(A B 3)$
$E 2=\exp \left(-T E / T_{2}\right)(A B 4)$

From equations 1 and 7, and equations 1 and 8, C can be expressed for the classical and spoiled gradient echo MRI protocols, respectively:

$$
C= \begin{cases}(1-E 1) \times E 2(9), \text { classical } & \\ & \text { MRI acquisition protocols. } \\ {[\sin F A \times(1-E 1) \times E 2] /(1-E 1 \cos F A)(10),} & \text { spoiled gradient echo }\end{cases}
$$

If a classical or spoiled gradient echo image acquisition protocol is used, then the expressions of $C$ in equations (9) and (10), respectively can be used to replace $\mathrm{C}$ in Equation 5.

Details of the texture analysis based algorithm (Talu, 2012) used for the f calculation are presented in Figure 1.

The $T_{1}$ and $T_{2}$ relaxation times for the $C / N$ and DA regions in Equation 5 can be calculated using the protocols presented in the literature (Deoni et al., 2005; Fanea and Sfrangeu, 2011). TR, TE, and FA in Equations 7 and 8 are known as the parameters set up in the MRI protocol for image acquisition. In MRI studies, a $\lambda$ value of $0.9 \mathrm{ml} / \mathrm{g}$ is usually used (Roberts et al., 1996). The f value can, then be calculated by simply measuring the signal intensities in the two corresponding regions in the $\mathrm{C} / \mathrm{N}$ and DA images (Figure 2). This allows the term $\Delta$ to be calculated as the difference between the signal intensity in the two corresponding regions. Then two more PD images of the C/N and DA subjects need to be acquired to assess $M_{0}$ and $\Delta_{0}$ in the two corresponding $C / N$ and DA regions. $M_{0}$ is calculated by simply measuring the signal intensity in the specific $\mathrm{C} / \mathrm{N}$ or DA region on the corresponding PD images. $\Delta_{0}$ represents the difference of the signal intensity measured on the $\mathrm{C} / \mathrm{N}$ and the corresponding DA regions on the PD images.

\section{DISCUSSION}

The potential of $\mathrm{MRI}$ for the non-invasive flow quantification in acquisition times of up to $1 \mathrm{~min}$ is demonstrated in this study using a theoretical mathematical model. The mathematical model for flow calculation can be applied offline, but future software developments will allow for flow quantification at the end of image acquisition. If flow charts are set up for $\mathrm{C} / \mathrm{N}$, then images need to be acquired only from the DA subjects. Even when the flow coefficients are calculated offline, these can give important information on the pathophysiology, together with the already clinically implemented multiparametric MRI techniques: relaxation 


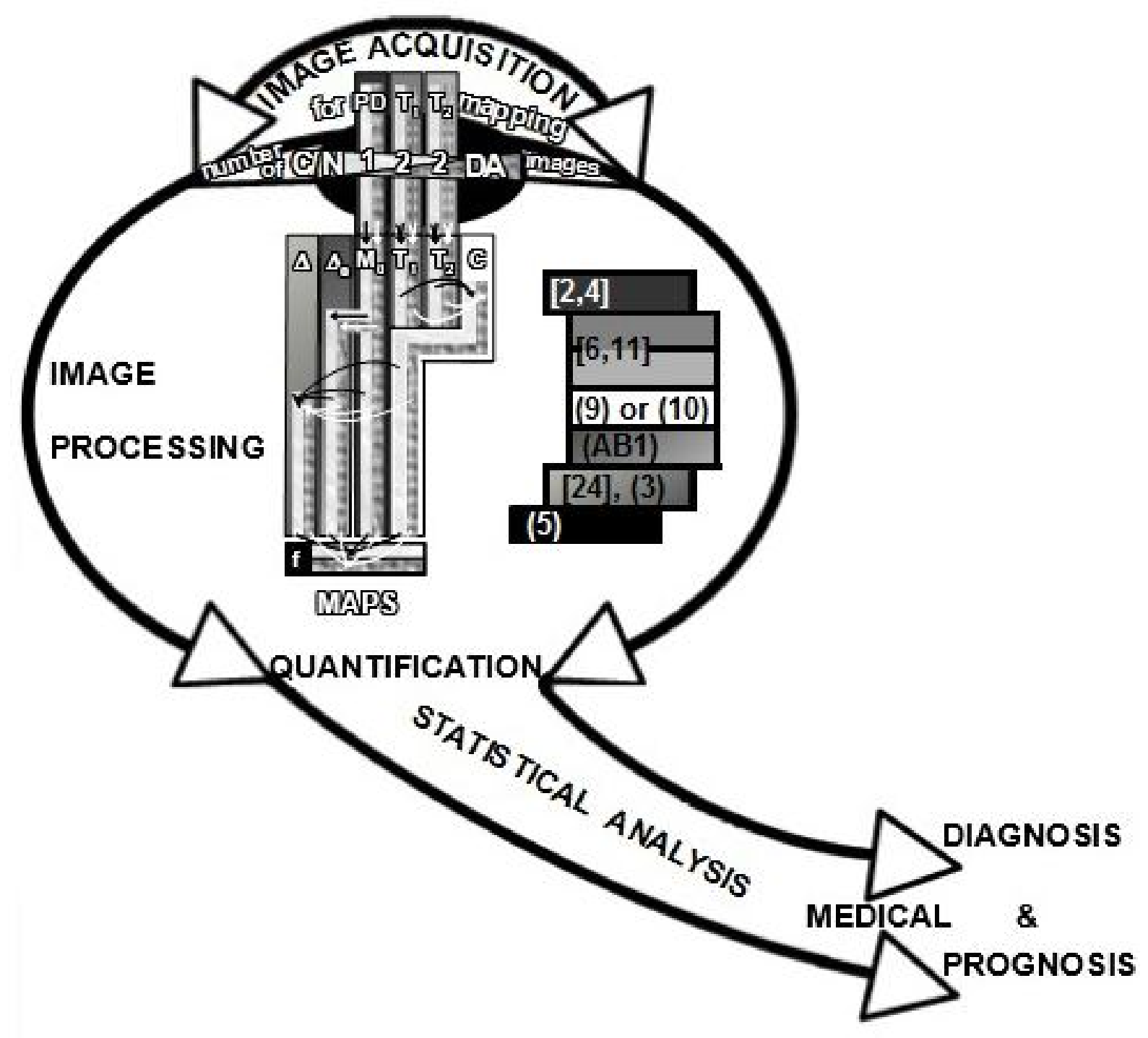

Figure 1. Texture analysis for flow quantification. Ten images: five of a $\mathrm{C} / \mathrm{N}$ and five of a $\mathrm{DA}$ subject are acquired for PD (Brown et al., 2014; Bernstein et al., 2004), $T_{1}$ and $T_{2}$ (Deoni et al., 2005; Fanea and Sfrangeu, 2011) mapping. If the $\mathrm{M}_{0}, \mathrm{~T}_{1}$ and $\mathrm{T}_{2}$ maps already exist, only two images need to be acquired: one from the $\mathrm{C} / \mathrm{N}$ and one from the $\mathrm{DA}$ subject. If a database for the $\mathrm{C} / \mathrm{N}$ already exists, then only the image of the $\mathrm{DA}$ subject needs to be acquired. The image(s) is(are) then processed and: $\Delta$ - (Roberts et al., 1996), Equation 3-, $\Delta_{0}$-abbreviation (AB1), $M_{0}-($ Brown et al., 2014; Bernstein et al., 2004)-, $T_{1}, T_{2}-$ (Deoni et al., 2005; Fanea and Sfrangeu, 2011)-, C -Equation 9 or 10- and f Equation 5 - maps of the $\mathrm{C} / \mathrm{N}$ and DA subjects are obtained. Flow is quantified in each region of interest and then statistically analysed based on Equations 3 and 5 for medical prognosis and diagnosis.

$[2,4]=[$ Bernstein et al. 2004, Brown et al. 2014]

$[6,11]=[$ Deoni et al. 2005, Fanea and Sfrangeu 2011]

$[24]=[$ Roberts et al. 1996]

times (Fanea and Fagan, 2012; Bojorquez et al., 2017), diffusion (Dong et al., 2004; Le Bihan, 2014), magnetization transfer ratios (Gupta, 2002; OrejaGuevara et al., 2006; Henkelman et al., 2001), magnetic susceptibility (Duyn, 2013; Wang and Liu, 2015; Liu et al., 2015), lengths (Fanea and Fagan, 2012; Feczko et al., 2009), areas (Feczko et al., 2009), volumes (Feczko et al., 2009), concentrations (Dong et al., 2004; Le Bihan, 2014; Gupta, 2002; Oreja-Guevara et al., 2006;
Henkelman et al., 2001; Duyn, 2013; Wang and Liu, 2015; Liu et al., 2015) and shear stiffnesses (Yin et al., 2016; Mariappan et al., 2010; Venkatesh and Ehman, 2015; Hiscox et al., 2016). The flow coefficients will contribute to the standard scale that can be built up for push-button automatic medical diagnosis. More importantly, introducing the flow quantification in the routine clinical MRI, detection of visually unperceived pathological changes could be possible, and therapies 


\section{Flow quantification using two MRI images acquired using exactly the same} imaging protocol from the following two subjects:

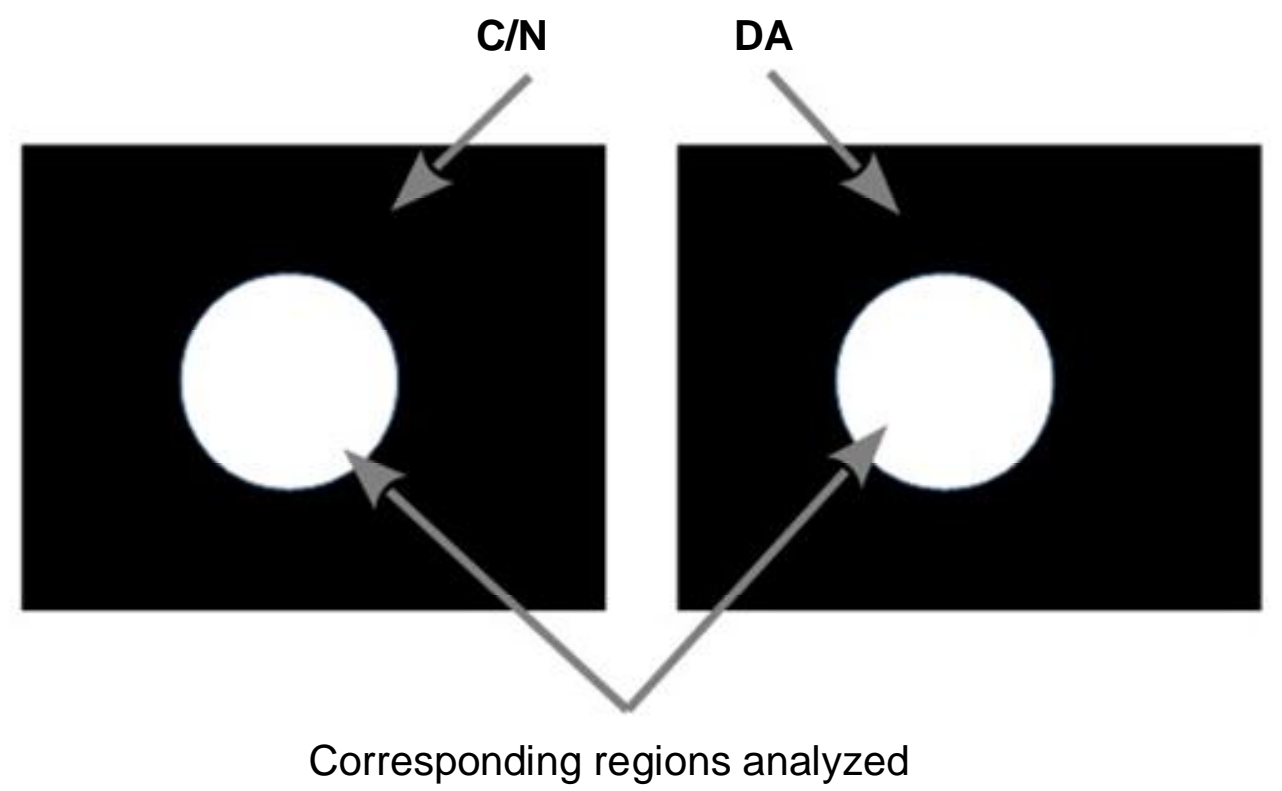

Figure 2. Flow assessment strategy relative to a $C / N$ region. $P D, T_{1}$ and $T_{2}$ maps from a $C / N$ and $D A$ subject are required for flow assessment in a region of interest (white circle). Acquisition of two more images (black squares), using exactly the same imaging protocol: one from a $\mathrm{C} / \mathrm{N}$ (left black square) and the other one from a DA (right black square) subject, is also required. Flow is then assessed from these maps and images using the texture analysis described in Figure 1.

could be applied immediately and more efficiently by the medical staff. This may contribute to the reduction of the existing socioeconomic burden caused by many diseases.

More studies are needed to: evaluate the clinical implementation of the method proposed in this study, build the $\mathrm{C} / \mathrm{N}$ flow standard scales, and assess the potential of this technique for disease detection, staging, assessment or for therapy and surgical evaluations.

\section{REFERENCES}

Amukotuwa SA, Yu C, Zaharchuk G, 2016. 3D pseudocontinuous arterial spin labeling in routine clinical practice: a review of clinically significant artifacts. J Magn Reson Imaging, 43(1): 11-27.

Bernstein MA, King KF, Zhou XJ, 2004. Handbook of MRI pulse sequences. Elsevier Academic Press, Burlington, San Diego, USA.

Bojorquez JZ, Bricq S, Acquitter C, Brunotte F, Walker PM, Lalande A, 2017. What are normal relaxation times of tissues at $3 \mathrm{~T}$ ? Magn Reson Imaging, 35: 69-80.

Brown RW, Cheng YCN, Haacke EM, Thompson MR, Venkatesan R, 2014. Magnetic Resonance Imaging: Physical Principles and Sequence Design, Second Edition. John Wiley \& Sons, Inc., Hoboken, New Jersey, USA

Calamante F, Thoams DL, Pell GS, Wiersma J, Turner R, 1999. Measuring cerebral blood flow using magnetic resonance imaging techniques. J Cerebral Blood Flow Metabol, 19: 701-735.

Deoni SC, Peters TM, Rutt BK, 2005. High-resolution $T_{1}$ and $T_{2}$ mapping of the brain in a clinically acceptable time with DESPOT1 and DESPOT2. Magn Reson Med, 53: 237-241.
Dong Q, Welsh RC, Chenevert TL, Carlos RC, Maly-Sundgren P, Gomez-Hassan DM, Mukherji SK, 2004. Clinical applications of diffusion tensor imaging. J Magn Reson Imaging, 19: 6-18.

Duyn, J 2013. MR Susceptibility Imaging. J Magn Reson. 229: 198207.

Fanea L, David LI, Lebovici A, Carbone F, Sfrangeu SA, 2012. Theoretical compartment modeling of DCE-MRI data based on the transport across physiological barriers in the brain. Comput Math Methods Med, 2012: 1-6.

Fanea L, Fagan AJ, 2012. Magnetic resonance imaging techniques in ophthalmology. Mol Vis 18: 2538-2536.

Fanea L, Sfrangeu SA, 2011. Relaxation times mapping using magnetic resonance imaging. Rom Rep Phys, 63: 456-464.

Feczko E, Augustinack JC, Fischl B, Dickerson BC, 2009. An MRIbased method for measuring volume, thickness and surface area of entorhinal, perirhinal, and posterior parahippocampal cortex. Neurobiol Aging, 30(3): 420-431.

Grade M, Hernandez Tamames JA, Pizzini FB, Achten E, Golay X, Smits M, 2015. A neuroradiologist's guide to arterial spin labeling MRI in clinical practice. Neuroradiology, 57: 1181-1202.

Gupta R, 2002. Magnetization transfer MR imaging in central nervous system infections. Ind J Radiol Imaging, 12(1): 51-58.

Haller S, Zaharchuk G, Thomas DL, Lovblad KO, Barkhof F, Golay X, 2016. Arterial spin labeling perfusion of the brain: emerging clinical application. Radiology, 281(2): 337-356.

Henkelman RM, Stanisz GJ, Graham SJ, 2001. Magnetization transfer in MRI: a review. NMR Biomed, 14: 57-64.

Herscovitch P, Raichle ME, 1985. What is the correct value for the brain-blood partition coefficient for water? J Cereb Blood Flow Metabol, 5: 65-69.

Hiscox LV, Johnson CL, Barnhill E, McGarry MDJ, Huston J, Beek EJR, Starr JM, Roberts N, 2016. Magnetic resonance elastography (MRE) of the human brain: technique, findings and clinical applications. Phys Med Biol, 61: 401-437. 
Le Bihan D, 2014. Diffusion MRI: what water tells us about the brain. EMBO Mol Med, 6(5): 569-573.

Liu C, Wei H, Gong NJ, Cronin M, Dibb R, Decker K, 2015. Quantitative susceptibility mapping: contrast mechanisms and clinical applications. Tomography, 1(1): 3-17.

Mariappan YK, Glaser KJ, Ehman RL, 2010. Magnetic resonance elastography: A review. Clin Anat, 23(5): 497-511.

Miller CG, Krasnow J, Schwartz LH, 2014. Medical Imaging in Clinical Trials. Springer-Verlag London, UK.

Oreja-Guevara C, Charil A, Caputo D, Cavarretta R, Sormani MP, Filippi $M$, 2006. Magnetization transfer magnetic resonance imaging and clinical changes in patients with relapsing-remitting multiple sclerosis. Arch Neurol 63: 736-740.

Roberts TPL, Vexler ZS, Vexler V, Derugin N, Kucharczyk I, 1996. Sensitivity of high-speed "perfusion-sensitive" magnetic resonance imaging to mild cerebral ischemia. Eur Radiol, 6: 645-649.

Soares DP, Law M, 2009. Magnetic resonance spectroscopy of the brain: review of metabolites and clinical applications. Clin Radiol, 64: 12-21.

Sourbrone SP, Buckley DL, 2013. Classic models for dynamic contrast-enhanced MRI. NMR Biomed, 26(8): 1004-1027.

Talu S, 2012. Texture analysis methods for the characterisation of biological and medical images. ELBA Bioflux, 4(1): 8-12.

Venkatesh SK, Ehman RL, 2015. Magnetic resonance elastography of abdomen. Abdomin Imaging, 40(4): 745-759.

Wang Y, Liu T, 2015. Quantitative susceptibility mapping (QSM): Decoding MRI data for a tissue magnetic biomarker. Magn Reson Med, 73: 82-101.

Yin M, Glaser KJ, Talwalkar JA, Chen J, Manduca A, Ehman RL, 2016. Hepatic Mr elastography: Clinical performance in a series of 1377 consecutive examinations. Radiology, 278: 114-124.
Citation: Fanea L, 2017. Theoretical mathematical model of noninvasive flow quantification for future implementation in routine clinical MRI. Int Res J Med Med Sci, 5(4): 58-63. 